\title{
PARTO NORMAL E CESÁREA: ESTUDO RETROSPECTIVO EM UMA MATERNIDADE ESCOLA
}

\section{NATURAL DELIVERY AND CESAREAN: A RETROSPECTIVE STUDY IN A SCHOOL MATERNITY}

\author{
Andressa Paola Ferreira ${ }^{1}$, Suellen Vienscoski Skupien ${ }^{1 *}$, Ana Paula Xavier Ravelli ${ }^{1}$ \\ ${ }^{1}$ Universidade Estadual de Ponta Grossa, Ponta Grossa, Paraná, Brasil \\ *Autor correspondente: Suellen Vienscoski Skupien. Universidade Estadual de Ponta Grossa \\ Av. General Carlos Cavalcanti, 4748, CEP 84030-900 - Ponta Grossa, Paraná, Brasil \\ E-mail: suvienscoski@gmail.com Telefone: (42) 999142927
}

\begin{abstract}
RESUMO
Estudo descritivo, com abordagem quantitativa, objetivou identificar os índices de parto normal e cesárea em uma maternidade escola referência ao parto de risco habitual/ intermediário da rede pública de saúde da cidade de Ponta Grossa, Paraná. Os dados advindos são do projeto de pesquisa "Consulta de Enfermagem no Pré-Natal e PósParto" da Universidade Estadual de Ponta Grossa. A amostra totalizou 1.745 puérperas entre o período de 2006 a 2014. Os resultados apontam que 53\% das puérperas possuem idade entre 21 e 30 anos; $40 \%$ encontram-se em união estável com o parceiro; $34 \%$ apresentam escolaridade superior a 12 anos de estudo e $66 \%$ não possuem vínculo empregatício. Em relação ao tipo de parto, $68 \%$ das puérperas tiveram parto normal e $32 \%$ cesárea. Portanto, conclui-se que a maternidade escola apresenta um índice de parto normal satisfatório, porém, as cesáreas ainda são elevadas, quando se considera os 15\% preconizados pela Organização Mundial da Saúde.
\end{abstract}

Palavras-chave: Parto normal; Cesárea; Saúde materna.

\begin{abstract}
This is a descriptive study, with a quantitative approach, aiming at identifying the rates of natural delivery and cesarean in a public normal/intermediated risk delivery referenced school maternity in Ponta Grossa, Paraná. Data comes from the Research Project "Consulta de Enfermagem no Pré-Natal e Pós Parto" from Universidade Estadual de Ponta Grossa. The sample gathered 1.745 puerperal women between 2006 and 2014. The results presented that $53 \%$ of the puerperal women are between 21 and 30 years; $40 \%$ are in a common-law marriage; $34 \%$ have studied for more than 12 years and $66 \%$ don't have an employment contract. Concerning to the delivery, $68 \%$ of the puerperal women had a natural delivery and $32 \%$ cesarean. Thus, we concluded that the school maternity presents a satisfactory rate of natural delivery. However, the numbers of cesareans are still high, considering the $15 \%$ recommended by the World Health Organizations.
\end{abstract}

Keywords: Natural delivery; Cesarean; Maternal health.

\section{INTRODUÇ̃̃O}

Define-se parto cesárea como uma intervenção cirúrgica que possibilita a retirada do bebê diretamente do útero materno ${ }^{(1)}$. Este tipo de procedimento surgiu com o intuito de salvar a vida da mãe e do feto em casos de complicações gravídicas, porém, a cesárea têm sido utilizada para satisfazer os desejos e as exigências da equipe médica e da gestante ${ }^{(2)}$.

O Brasil é um dos países que se destacam perante a realização do parto cesárea, os índices deste 
procedimento assumiram proporções espantosas e crescem cada vez mais. Conforme dados do Ministério da Saúde, em 2010, 52\% dos partos no País foram cirúrgicos. Na rede privada o índice chega a $82 \%$ e, na pública, onde ocorre $3 / 4$ de todos os partos, são $37 \% \%^{(3)}$. Estudo realizado pela Agência Nacional de Saúde Suplementar, em 2015, apontou que o número de parto cesárea chegava a $84 \%$ na rede privada, enquanto na rede pública de saúde atingia 40\%. Esses dados reforçam a ressalva feita pela Organização Mundial da Saúde (OMS) que considera epidêmica a elevação dos índices de cesárea, com valores que superem os $15 \%$ preconizados $^{(4,5)}$.

A cesárea é indicada quando ocorrem apresentações anômalas do feto, em casos de doenças crônicas, como diabetes gestacional e hipertensão arterial ou ainda: doenças sexualmente transmissíveis; descolamento prematuro de placenta; placenta prévia; excessos ou escassez de líquido amniótico; trabalho de parto prematuro; incompatibilidade $\mathrm{Rh}$; atonia uterina; dentre outros fatores capazes de gerar complicações no andamento do parto. Assim, a cesárea é um método cirúrgico, que concede um nascimento sem maiores inconveniências e dificuldades, sendo que este método só deverá ser executado se houver riscos à vida da mãe ou da criança, servindo, portanto, como um meio de segurança e proteção a saúde materno-infantil ${ }^{(2,6)}$.

$\mathrm{O}$ modelo de assistência ao parto tem-se fundamentado cada dia mais em técnicas e métodos cirúrgicos $^{(6)}$. A escolha da cesárea, por parte da mulher, está associada ao medo da dor e também pela influência médica, pois alguns profissionais omitem as informações sobre os riscos e contraindicações desse procedimento, ou ainda, prescrevem sua realização sem necessidade durante o pré-natal ou trabalho de $\operatorname{parto}^{(7,8)}$.

O parto normal, por sua vez, consiste no nascimento do bebê através do colo do útero e canal vaginal. Neste tipo de parto a recuperação da mulher é imediata, pois, logo após o nascimento ela se torna independente, conseguindo realizar seus cuidados e os do recém-nascido, ou seja, a recuperação é mais rápida, há menor sensação de dor no período pós-parto, a alta é precoce, há um menor risco de infecção e de hemorragia. Salienta-se que as complicações inerentes ao parto normal são de menor gravidade no momento em que se equipara com as complicações que surgem perante o parto cesáreo ${ }^{(8)}$.
A gratificação da mulher após o parto normal é descrita por sentimentos positivos, muitas relatam a experiência como sendo maravilhosa e gratificante, uma vez que a mulher se torna a personagem principal daquele determinado momento e os profissionais, por sua vez, estão ali somente para ampará-la $a^{(7,9)}$.

Diversos estudos ${ }^{(9,10,11)}$ apontam que um alto índice de mulheres tem preferência pelo parto normal, ou seja, cerca de 70 a $80 \%$ das mulheres da rede pública e do serviço privado possuem esta preferência. Dentre os itens de escolha para este tipo de parto estão as vantagens especialmente de recuperação rápida perante o parto cesáreo, por este apresentar maiores dificuldades no pós-operatório ${ }^{(2,8)}$.

A OMS preconiza que, para segurança da mãe e do filho, o processo de nascimento sofra o mínimo de intervenções, respeitando-se, portanto, o processo fisiológico do nascimento ${ }^{(4)}$. Porém, como o trabalho de parto demanda, na maioria das vezes, muito mais horas do que a realização de um parto cesárea, quase sempre ocorre intervenção na tentativa de regulação e controle do parto pelos médicos ${ }^{(2)}$.

Atualmente, um dos maiores desafios do Ministério da Saúde é a reversão dos dados estatísticos referentes ao parto cesáreo no Brasil. Muitas estratégias surgem com o intuito de incentivar o parto normal e usa humanização, como: estímulo para a atuação da enfermeira obstétrica; utilização de práticas baseadas em evidências científicas; acesso às tecnologias apropriadas de atenção ao parto; presença de acompanhante durante o trabalho de parto, parto e pós-parto; posição de parto de escolha pela mulher; e as políticas públicas ${ }^{(12-14)}$.

O Ministério da Saúde traz políticas que defendem a saúde da mulher como o Programa de Assistência Integral à Saúde da Mulher (PAISM), que engloba questões de cunho materno-infantil da concepção à anticoncepção, vida social e sexual, entre outros $^{(12)}$. A Rede Cegonha é outra política voltada ao ciclo gravídico-puerperal implantada com o objetivo de umamelhor qualificação no atendimento a gestantes, parturientes e puérperas ${ }^{(13)}$.

Há ainda documentos lançados recentemente pelo Ministério da Saúde como o Caderno Humaniza SUS, que aborda uma ampla discussão sobre a humanização do parto e do nascimento, garantindo acesso com qualidade e mencionando a inclusão do enfermeiro obstetra na realização dos partos de risco 
habitual. Este documento ainda menciona a mulher como protagonista do parto, portanto, nesse aspecto os profissionais devem respeitar o processo fisiológico da mulher deixando de intervir desnecessariamente ${ }^{(14)}$.

Dentro desta análise de políticas de humanização, o Ministério da Saúde lançou ainda o Caderno de Diretrizes (2013-2015), que visa organizar a Rede de Atenção Materno-infantil, sendo uma de suas metas aumentar o percentual de partos normais no País, garantindo acolhimento adequado e resolutivo ${ }^{(15)}$.

O Caderno de Atenção Básica no 32 "Atenção ao Pré-natal de Baixo Risco" aborda a assistência pré-natal prestada pelos serviços de atenção primária à saúde, mencionando o fato desses se tornarem qualificados e adequados às demandas ${ }^{(16)}$. Assim, os profissionais das Unidades de Saúde devem preparar e incentivar as mulheres, desde o início da gestação, ao parto normal, de forma que aprendam a controlar a dor para não optarem pelo parto cesáreo, incluindo também orientações sobre o momento mais sensato para a internação hospitalar mediante ao trabalho de parto, prevenindo, assim, a hospitalização precoce, indicada como uma das causas para o crescimento dos índices de parto cesárea ${ }^{(1,8)}$.

No Paraná existe, ainda a Rede Mãe-Paranaense, que possuí suas ações voltadas à atenção primária de saúde, sendo sua política eficiente na redução da morbimortalidade materno-infantil. Isso se deve ao fato do fornecimento de um pré-natal de qualidade em que se classifica a mãe e o recém-nascido, conforme suas complexidades, vinculando-os a uma maternidade de referência que possuí estrutura adequada para a realização de um atendimento qualificado e especializado $^{(17)}$.

Diante deste contexto de políticas públicas, a Agência Nacional de Saúde Suplementar instituiu, em janeiro de 2015, a Resolução Normativa n 368 , que estabelece a obrigatoriedade dos percentuais de cirurgias cesáreas e de parto normais, por operadora, por estabelecimento de saúde e por médico, objetivando incentivar o parto normal ${ }^{(5)}$.

Além dos documentos elaborados pelo Ministério da Saúde, existem estudos ${ }^{(18,19)}$ que propõem outras estratégias para que haja redução do número de partocesáreo, tais como: mudanças na formação profissional com vistas à superação do modelo tecnicista, apoiando o modelo multiprofissional; inserção de diferentes profissionais capacitados, desmistifica o fato de que o parto deve ser realizado somente pelo profissional médico, inserindo, nesse cenário, profissões que possuem um enfoque maior na humanização, no processo fisiológico e na educação em saúde.

Dessa forma a questão que direciona este estudo é: "mediante ao projeto Consulta de Enfermagem no Pré-Natal e Pós-Parto (CEPP) é possível identificar o índice de parto cesárea"?

Assim, este estudo objetivou identificar os índices de parto normal e cesárea em uma maternidade escola, referência ao parto de risco habitual/intermediário, no município de Ponta Grossa, Paraná.

\section{METODOLOGIA}

Este estudo caracteriza-se como descritivo, fundamentado na abordagem quantitativa. Os dados aqui utilizados foram fornecidos pelo projeto de extensão e pesquisa "Consulta de

Enfermagem no Pré-Natal e Pós-Parto (CEPP)", da Universidade Estadual de Ponta Grossa (UEPG), aprovado sob o parecer número 1.055.927 e expedido pelo Comitê de Ética em Pesquisa da referida Universidade.

O estudo foi realizado em uma maternidade escola, referência ao parto de risco habitual/ intermediário, da rede pública de saúde, do município de Ponta Grossa, Paraná. A população foi composta por mulheres em puerpério imediato $\left(1^{\circ}\right.$ ao $10^{\circ}$ dia $)$, as quais concordaram em participar da entrevista assinando o Termo de Consentimento Livre e Esclarecido (TCLE).

Trata-se de uma amostra por conveniênciacom 1.745 puérperas, entre os anos de 2006 a 2014. As entrevistas foram realizadas por acadêmicos do $3^{\circ} \mathrm{e}$ $4^{\circ}$ anos do curso de Bacharelado em Enfermagem da Universidade Estadual de Ponta Grossa (UEPG), os quais, após ministrarem palestras voltadas à saúde materno-infantil, solicitaram a participação voluntária das puérperas na pesquisa.

O instrumento de coletade dados consistiu de um questionário estruturado, contendo perguntas referentes a: identificação, antecedentes ginecológicos, obstétricos e familiares, estado nutricional e gestação atual. Os dados foram organizados em planilhas do Excel e expressos em frequências simples. 


\section{RESULTADOS}

Em relação ao perfil socioeconômico, apresentado na Tabela 1 , verificou-se que $53 \%(n=926)$ das puérperas estavam entre a faixa etária de 21 a 30 anos. Com relação ao estado civil, $40 \%(n=700)$ se encontravam em união estável com o parceiro. No grau escolar destaca-se que $27 \%(\mathrm{n}=473)$ possuíam ensino médio completo; enquanto vínculo empregatício, um índice de $66 \%(\mathrm{n}=1156)$ relatou não o possuir.
Ressalta-se, ainda, na Tabela 1, o ano de 2012 com maior número de entrevistadas, somando 281 puérperas e o ano de 2011, com menor número de entrevistadas, totalizando apenas 35 participantes. Este viés de 2011 se deve a uma menor participação por parte dos acadêmicos no "Projeto Consulta de Enfermagem no Pré-Natal e Pós-Parto (CEPP)". Apesar deste viés importante, totalizamos uma amostra de 1.745 puérperas, com uma média de 176 entrevistadas por ano.

Tabela1 -Representação do perfil socioeconômico das puérperas entrevistadas na maternidade escola. Ponta Grossa, PR, Brasil, 2006-2014

\begin{tabular}{|c|c|c|c|c|c|c|c|c|c|c|c|c|c|c|c|c|c|c|c|c|}
\hline & \multirow{2}{*}{\begin{tabular}{|l}
2006 \\
$\mathrm{n}$ \\
\end{tabular}} & \multirow[b]{2}{*}{$\%$} & \multirow{2}{*}{\begin{tabular}{l|}
2007 \\
$n$
\end{tabular}} & \multirow[b]{2}{*}{$\%$} & \multirow{2}{*}{\begin{tabular}{|l|}
2008 \\
$\mathrm{n}$ \\
\end{tabular}} & \multirow[b]{2}{*}{$\%$} & \multirow{2}{*}{\begin{tabular}{|l|}
2009 \\
$\mathrm{n}$ \\
\end{tabular}} & \multirow[b]{2}{*}{$\%$} & \multirow{2}{*}{$\begin{array}{l}2010 \\
\mathrm{n}\end{array}$} & \multirow[b]{2}{*}{$\%$} & \multirow{2}{*}{$\begin{array}{l}2011 \\
\mathrm{n}\end{array}$} & \multirow[b]{2}{*}{$\%$} & \multirow{2}{*}{\begin{tabular}{|l|}
2012 \\
$n$
\end{tabular}} & \multirow[b]{2}{*}{$\%$} & \multirow{2}{*}{$\begin{array}{l}2013 \\
\mathrm{n} \\
\end{array}$} & \multirow[b]{2}{*}{$\%$} & \multirow{2}{*}{\begin{tabular}{|l|}
2014 \\
$\mathrm{n}$ \\
\end{tabular}} & \multirow[b]{2}{*}{$\%$} & \multicolumn{2}{|c|}{\begin{tabular}{|c|} 
2006-20014 \\
(TOTAL)
\end{tabular}} \\
\hline & & & & & & & & & & & & & & & & & & & $\mathrm{n}$ & $\%$ \\
\hline Total & 251 & 14 & 240 & 14 & 263 & 15 & 180 & 10 & 148 & 9 & 35 & 2 & 281 & 16 & 180 & 10 & 167 & 10 & 1.745 & 100 \\
\hline \multicolumn{21}{|l|}{ Idade Materna } \\
\hline$\leq 20$ anos & 63 & 25 & 61 & 25 & 66 & 25 & 51 & 28 & 40 & 27 & 7 & 20 & 78 & 28 & 51 & 29 & 64 & 39 & 481 & 28 \\
\hline 21 a 30 anos & 148 & 59 & 131 & 55 & 145 & 55 & 88 & 49 & 82 & 55 & 18 & 51 & 158 & 56 & 88 & 49 & 68 & 41 & 925 & 53 \\
\hline$>31$ anos & 40 & 16 & 48 & 20 & 52 & 20 & 41 & 23 & 26 & 18 & 10 & 29 & 45 & 16 & 41 & 22 & 35 & 20 & 338 & 19 \\
\hline \multicolumn{21}{|l|}{ Estado Civil } \\
\hline Solteira & 44 & 17 & 44 & 19 & 55 & 18 & 36 & 20 & 27 & 18 & 8 & 23 & 56 & 20 & 36 & 20 & 44 & 27 & 350 & 20 \\
\hline Casada & 120 & 48 & 96 & 40 & 102 & 40 & 60 & 33 & 71 & 48 & 9 & 28 & 85 & 30 & 60 & 33 & 68 & 40 & 671 & 38 \\
\hline União estável & 80 & 32 & 96 & 40 & 102 & 40 & 81 & 45 & 50 & 34 & 17 & 48 & 138 & 49 & 81 & 45 & 55 & 33 & 700 & 40 \\
\hline \multicolumn{21}{|l|}{ Grau Escolar } \\
\hline $\begin{array}{l}\text { Fundamental } \\
\text { Incompleto }\end{array}$ & 74 & 30 & 29 & 12 & 31 & 12 & 64 & 36 & 31 & 21 & 7 & 20 & 70 & 25 & 56 & 31 & 45 & 33 & 407 & 23 \\
\hline $\begin{array}{l}\text { Fundamental } \\
\text { Completo }\end{array}$ & 65 & 26 & 81 & 34 & 90 & 34 & 30 & 17 & 33 & 22 & 9 & 26 & 40 & 14 & 22 & 12 & 18 & 11 & 388 & 22 \\
\hline $\begin{array}{l}\text { Médio } \\
\text { Incompleto }\end{array}$ & 39 & 15 & 50 & 21 & 55 & 21 & 35 & 19 & 26 & 18 & 6 & 17 & 64 & 23 & 27 & 15 & 37 & 22 & 339 & 19 \\
\hline Médio Completo & 61 & 24 & 68 & 28 & 74 & 28 & 38 & 21 & 50 & 34 & 8 & 23 & 89 & 32 & 30 & 17 & 55 & 27 & 473 & 27 \\
\hline Superior & 12 & 5 & 12 & 5 & 13 & 5 & 13 & 7 & 8 & 5 & 5 & 14 & 18 & 6 & 5 & 3 & 12 & 7 & 98 & 7 \\
\hline \multicolumn{21}{|l|}{$\begin{array}{l}\text { Vinculo } \\
\text { Empregatício }\end{array}$} \\
\hline Sim & 76 & 30 & 83 & 35 & 92 & 35 & 51 & 28 & 90 & 61 & 13 & 37 & 82 & 29 & 51 & 28 & 38 & 23 & 576 & 33 \\
\hline Não & 175 & 70 & 157 & 65 & 171 & 65 & 129 & 72 & 58 & 39 & 22 & 63 & 199 & 71 & 128 & 71 & 117 & 69 & 1.156 & 66 \\
\hline
\end{tabular}

Fonte: Projeto CEPP

Em relação ao tipo de parto realizado na maternidade escola referência ao parto de risco habitual/intermediário destaca-se o período de 2006 a 2014 de uma maneira geral, quando $68 \%(n=1.194)$ dos partos foram normais e $32 \%(n=551)$ cesáreas.

\section{DISCUSSÃO}

Em relação ao perfil socioeconômico e tipo de parto, nesse estudo, teve-se, majoritariamente, mulheres na faixa etária de 21 a 30 anos; dessas $27 \%$ tinham ensino médio completo e tiveram parto normal, contrapondo-se a um estudo ${ }^{(20)}$ realizado com mulheres com idade mais avançada, escolaridade superior a 12 anos de estudo e com experiência reprodutiva anterior optando pelo parto cesárea.

Outro estudo $^{(9)}$, realizado em 2013, demonstra que a idade influenciou de forma significativa na escolha do parto, segundo o autor a escolha pela 
cesárea é proporcional ao amadurecimento da mulher, ou seja, quanto maior a idade da mulher, maior será a probabilidade de escolha pela cesárea. Destacou-se, ainda, nesse estudo que mulheres em união estável ou casadas possuíam preferência pelo parto normal, enquanto as solteiras manifestam interesse pelo parto cesárea.

Sendo assim, na maternidade escolade Ponta Grossa encontramos um índice significativo de mulheres com idade superior a 31 anos, atingindo $19 \%(\mathrm{n}=338)$ e um índice significativo de puérperas em união estável ou casadas com $78 \%(\mathrm{n}=1.371)$. Estes dados somados de forma conjunta com outros fatores socioeconômicos justificam a escolha pelo parto cesárea, taxa que se demonstra significativa no estudo.

Observou-se, por meio de estudo ${ }^{(21)}$, que a escolaridade influencia na escolha do parto, ou seja, mulheres com grau maior de instrução possuem preferência pelo parto cesárea. $\mathrm{O}$ autor ainda relata que as mulheres justificam essa escolha pelo fato de poder optar pela data/hora da realização do procedimento, sem comprometer as atividades profissionais.

Ressalta-se, também, que apesar da escolaridade influenciar no tipo de parto, muitas mulheres iniciam o pré-natal com o desejo de realizar o parto normal, porém, à medida que realizam as consultas, esse desejo é substituído pela cesárea, o que reforça a influência e importância dos profissionais de saúde para auxiliarem a gestante na escolha do tipo de parto ${ }^{(21)}$.

Estudo $^{(20)}$ demonstra que o nível social não possuí influência direta na escolha de parto, segundo seu autor, a escolha pela cesárea está atrelada à crença de que um procedimento tecnicista é mais seguro que $\mathrm{o}$ andamento fisiológico do processo.

No Brasil, as taxas de cesárea são elevadas, em torno de $35 \%$, e atingem valores ainda maiores nos serviços privados, chegando a cerca de 70 a $90 \%$ dos partos realizados ${ }^{(20)}$. Deve-se reforçar, aqui, que a OMS considera epidêmica a elevação das taxas de cesárea, com valores que superam os $15 \%$ preconizados ${ }^{(4)}$.

$\mathrm{Na}$ maternidade referência em partos de risco habitual/intermediário verificou-se que, durante o período de 2006 a 2014, houve a realização de 1.194 partos normais, o que representa $68 \%$ da amostra, enquanto que a cesárea foi realizada em 551 mulheres, representando $32 \%$. O número de cesáreas que poderiam ser realizados mediante a taxa de $15 \%$ preconizada pela OMS para a amostra de 1.745 puérperas seria de apenas 262 procedimentos, porém, houve a realização de 289 procedimentos a mais, o que expõe as mulheres a um risco considerável.

Salienta-se que a cesárea deve ser realizada somente quando há riscos de complicações na saúde da mãe e do feto ${ }^{(22)}$. A OMS preconiza que a assistência ao nascimento sofra o mínimo de intervenções possíveis, resgatando-se, assim, a valorização do processo fisiológico ${ }^{(4)}$.

No Brasil, a atenção ao parto é considerada uma das piores do mundo, pois ocorrem mortes por ausência de tecnologia adequada ou pela aplicação exagerada de tecnologia inapropriada ${ }^{(6,22)}$.

O Ministério da Saúde tem desenvolvido estratégias para promover um novo modelo de assistência que vise à humanização e à redução das intervenções desnecessárias, tendo em vista que há um excesso de cesáreas sendo realizadas, gerando problemas na saúde pública, riscos à saúde maternoinfantil e aumento no custo do financiamento público de saúde ${ }^{(6)}$.

\section{CONCLUSÃO}

Evidencia-se a necessidade da continuidade na investigação dos fatores que promovem o aumento de cesáreas na maternidade escola, uma vez que podem ocasionar uma série de complicações, tanto para as mães quanto para seus filhos. Além disso, os índices encontram-se muito acima do recomendado pela OMS, demonstrando que políticas públicas dirigidas à redução das taxas de cesarianas são urgentes e necessárias.

O projeto "Consulta de Enfermagem no Pré-natal e Pós-parto" trabalha em parceria com a maternidade escola no intuito de melhorar o atendimento às parturientes e puérperas. $\mathrm{O}$ objetivo do projeto é fornecer educação em saúde a esse grupo, incentivando o aleitamento materno e esclarecendo dúvidas em relação a temas pertinentes à saúde da mulher, que incluem temas relacionados ao recémnascido, planejamento familiar e, por vezes, parto e puerpério. A maternidade escola encontra-se em processo de implantação do parto humanizado, porém, a jornada ainda é longa até que se instale essa proposta e o atendimento se torne qualificado, garantindo às gestantes a escolha pelo tipo de parto mais adequado a cada situação. 


\section{REFERÊNCIAS}

1. Queiroz MVO, Silva NSJ, Jorge MSB, Moreira TMM. Incidência e Características de Cesáreas e de Partos Normais: Estudo em uma Cidade no Interior do Ceará. Rev Bras Enferm. [Internet] 2005;58(6) [acesso em 21 mar 2015]. Disponível: http://www.scielo.br/scielo.php?script=sci_artt ext\&pid=S0034-71672005000600011

2. Chiavegatto Filho ADP. Partos Cesáreos e a Escolha a Data de Nascimento no Município de São Paulo. Ciência \& Saúde Coletiva. [Internet] 2013;18(8) [acesso em 21 mar 2015]. Disponível: http://www.scielo.br/scielo. php?pid=S1413-81232013000800026\&script=sci_arttext

3. Brasil. Ministério da Saúde. Canal Saúde: Construindo Cidadania. Fiocruz Pesquisa Preferência das Brasileiras pela Cesariana. [Internet] 2015 [acesso em 04 nov 2015]. Disponível: http://www.canal.fiocruz.br/destaque/index. php?id $=450$

4. Organização Mundial da Saúde. Declaração da OMS sobre Taxas de Cesárea. [Internet] [acesso em 04 nov 2015]. Disponível: http://apps.who.int/iris/ bitstream/10665/161442/3/WHO_RHR_15.02_por.pdf

5. Brasil. Agencia Nacional de Saúde Suplementar. ANS Publica Resolução para Estimular Parto Normal na Saúde Suplementar. [Internet] 2015 [acesso em 29 jul 2015]. Disponível: http://www.ans.gov.br/aans/noticias-ans/ consumidor/2718-ministerio-da-saude-e-ans-publicamresolucao-para-estimular-parto-normal-na-saudesuplementar

6. Vieira ABL, Lima VER. Crescente Incidência de Partos Cesáreos no Brasil. In. Ciegesi. $1^{\text {a }}$ Conferência Internacional De Estratégia Em Gestão, Educação E Sistemas De Informação. Goiânia, Go, Brasil. [Internet] 2012 [acesso em 21 mar 2015]. Disponível:

https://www.google.com.br/search?q=Crescente + Incid $\% \mathrm{C} 3$ $\%$ AAncia + De + Partos + Ces\%C3\%A1 reos + No + Brasil. + In. + Ciegesi.\&oq $=$ Crescente + Incid $\%$ C3\%AAncia + De + Partos + Ces\%C3\%A1 reos + No + Brasil. + In. + Ciegesi.\&aqs $=$ chrome ..69i57.874j0j4\&sourceid $=$ chrome\&es_sm $=93 \& \mathrm{ie}=\mathrm{UTF}-8$

7. Velho MB, Santos EKA, Collaço VS. Parto Normal e Cesárea: Representações Sociais de Mulheres que os Vivenciaram. Rev Bras Enferm. [Internet] 2014; 67(2) [acesso em 21 mar 2015]. Disponível: http://www.redalyc. org/articulo.oa? $\mathrm{id}=267030687017$

8. Domingues RMSM, et al. Processo de Decisão pelo Tipo de Parto do Brasil: Da Preferência Inicial das Mulheres à Via de Parto Final. Cad. Saúde Pública. [Internet] 2014 [acesso em 21 mar 2015]. Disponível: http://www.scielo.br/ pdf/csp/v30s1/0102-311X-csp-30-s1-0101.pdf

9. Leguizamon Junior T, Steffani JA, Bonamigo EL. Escolha da Via de Parto: Expectativa de Gestantes e Obstetras.Rev. Bioét. [Internet] 2013;21(3) [acesso em 21 mar 2015].
Disponível: http://revistabioetica.cfm.org.br/index.php/ revista_bioetica/article/viewFile/798/934

10. Lamarca G, Vettore M. Cesarianas No Brasil: Uma Preferência das Gestantes ou dos Médicos? Determinantes Sociais da Saúde: Portal e observatório sobre iniquidades em Saúde. [Internet] 2012 [acesso em 21 mar 2015]. Disponível: http://dssbr.org/site/2012/12/cesarianas-nobrasil-uma-preferencia-das-gestantes-ou-dos-medicos/

11. Matos GC, Escobal AP, Soares MC, Harter J, Gonzales RIC. A Trajetória Histórica das Políticas de Atenção ao Parto no Brasil: Uma Revisão Integrativa. Rev Enferm. UFPE. [Internet] 2013;7(esp) [acesso em 21 mar 2015]. Disponível: https://www.google.com.br/\#q=A+Trajet $\% \mathrm{C} 3$ $\% \mathrm{~B} 3$ ria + Hist $\% \mathrm{C} 3 \% \mathrm{~B} 3$ rica + Das + Pol $\% \mathrm{C} 3 \%$ ADticas + De + Aten $\% \mathrm{C} 3 \% \mathrm{~A} 7 \% \mathrm{C} 3 \% \mathrm{~A} 3 \mathrm{o}+\mathrm{Ao}+\mathrm{Parto}+\mathrm{No}+\mathrm{Brasil}:+\mathrm{Uma}+\mathrm{R}$ evis $\% \mathrm{C} 3 \% \mathrm{~A} 3 \mathrm{o}+$ Integrativa

12. Brasil. Ministério da Saúde. Política Nacional de Atenção Integral à Saúde da Mulher. [Internet] 2004 [acesso em 04 nov 2015]. Disponível: http://bvsms.saude.gov.br/ bvs/publicacoes/politica_nac_atencao_mulher.pdf

13. Brasil. Ministério da Saúde. Rede Cegonha. [Internet] 2013 [acesso em 04 nov 2015]. Disponível: http://bvsms. saude.gov.br/bvs/folder/rede_cegonha.pdf

14. Brasil. Ministério da Saúde. Secretária de Atenção Básica á Saúde. Política Nacional de

Humanização. Cadernos Humaniza SUS - Humanização do Parto e Nascimento.Vol. 4. Informes Técnicos: Cadernos de Saúde. Brasília. [Internet] 2014 [acesso em 21 mar 2015]. Disponível: http://bvsms.saude.gov.br/bvs/publicacoes/ caderno_humanizasus_v4_humanizacao_parto.pdf

15. Brasil. Ministério da Saúde. Secretária de Gestão Estratégica e Participativa. Departamento de Articulação Interfederativa. Caderno de Diretrizes - objetivos, metas e indicadores 2013-2015. Informes Técnicos: Cadernos de Saúde. [Internet] 2013 [acesso em $21 \mathrm{mar}$ 2015]. Disponível: http://189.28.128.100/sispacto/ CadernoDiretrizes2013_2015.pdf

16. Brasil. Ministério da Saúde. Secretária de Atenção Básica. Departamento de Atenção Básica. Cadernos de Atenção Básica - Atenção ao Pré-natal de Baixo Risco. Informes Técnicos: Cadernos de Saúde. Brasília. [Internet] 2012 [acesso em 21 mar 2015]. Disponível: http:// bvsms.saude.gov.br/bvs/publicacoes/cadernos_atencao_ basica_32_prenatal.pdf

17. Paraná. Secretária de Saúde do Paraná. Linha Guia Mãe Paranaense. Informes Técnicos: manual de orientação. Paraná. [Internet] 2012. [acesso em 21.out.2015]. Disponível em: http://www.saude.pr.gov.br/arquivos/File/ACS/linha guia_versao_final.pdf

18. Mouta RJO, Progianti JM. Estratégias de Luta das Enfermeiras da Maternidade Leila Diniz para Implantação 
de um Modelo Humanizado de Assistência ao Parto. Texto Contexto Enferm. [Internet] 2009;18(4) [acesso em 21 mar 2015]. Disponível: http://www.scielo.br/scielo. php?script=sci_arttext\&pid=S0104-07072009000400015

19. Vogt SE, et al. Características da Assistência ao Trabalho de Parto e Parto em Três Modelos de Atenção no SUS, No Município De Belo Horizonte, Minas Gerais, Brasil. Cad. Saúde Pública. [Internet] 2011;27(9) [acesso em 21 mar 2015]. Disponível: http://www.scielo.br/scielo. php?script=sci arttext\&pid=S0102-311X2011000900012

20. Dias MAB, et al. Trajetória das Mulheres na Definição pelo Parto Cesáreo: Estudo de Caso em Duas Unidades do Sistema de Saúde Suplementar do Estado do Rio De Janeiro. Ciência \& Saúde Coletiva. [Internet] 2008;13(5) [acesso em 21 mar 2015]. Disponível: http://www.scielo.br/scielo. php?script=sci_arttext\&pid=S1413-81232008000500017

21. Pires D, Fertonani HP, Conill EM, Matos TA, Cordova F, Mazur CS. A Influência da

Assistência Profissional em Saúde na Escolha do Tipo de Parto: Um Olhar Sócio Antropológico na Saúde Suplementar Brasileira. Rev. Bras. Saúde Matern. Infant. [Internet] 2010;10(2) [acesso em 29 jul 2015]. Disponível:http://www.scielo.br/scielo.php?pid=S151938292010000200006\&script=sci_arttext

22. Leão MRC, Riesco MLG, Schneck CA, Angelo M. Reflexões Sobre o Excesso de Cesarianas no Brasil e a Autonomia das Mulheres.Ciência \& Saúde Coletiva. [Internet] 2013;18(8) [acesso em 21 mar 2015]. Disponível: http://www.producao.usp.br/bitstream/handle/BDPI/46310/ Reflex $\%$ C3\%B5es\%20sobre $\% 20$ o $\% 20$ excesso $\% 20$ de $\% 20$ cesarianas $\% 20$ no $\% 20$ Brasil $\% 20 \mathrm{e} \% 20 \mathrm{a} \% 20$ autonomia $\% 20$ das $\% 20$ mulheres.pdf? sequence $=1 \&$ isAllowed $=y$ 